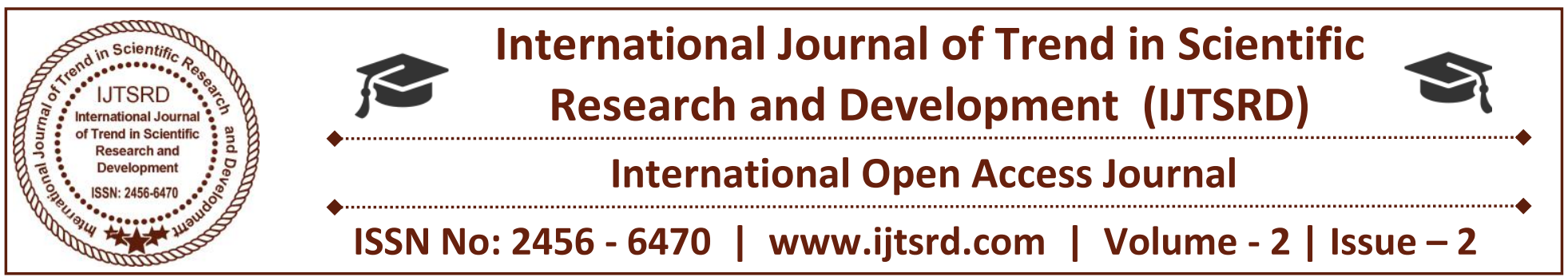

\title{
Community health concern and behavioral sciences
}

\author{
Dr Veena. S. Algur \\ Faculty, Department of Community Medicine, BLDE University's \\ Shri B M Patil Medical College, Hospital and Research Center Bijapur, Karnataka, India
}

\section{ABSTRACT}

Now it is a wake up time to India to fight against all odds and move towards health for all by reorienting existing priorities in health care services from curative to preventive on the basis of felt need services to the community. Innovative, need based ,slow and steady race will definitely lead us to "Health for all" A developing country like India's, hurdles of development are various and vicious in nature viz burning social problems like poverty, illiteracy, ignorance, conservatism, un-employment, gender bias etc such social, political and cultural factors have led to a number of multifaceted and complex health problems. Among all the developmental issues health for all is a mirage health care, health promotion has to be given a special priority .Mere existence of health services is not enough there is need to focus on utilization of available health care facility which is based on the awareness of general public and influencing socioeconomic and socio cultural factors like literacy, economic condition cultural practices and gender, prevalent in community.

Keywords: Community Health Care, Behavioral science, Health Services

\section{Introduction}

People mostly contact infection in the community and not in the hospital . They come to hospital for treatment so if we are really committed to curb the mortality and morbidity. We must understand the community in depth and the different forces operating in it, which can only be done if we have a sufficient understanding of the subject matters of behavioral sciences. However an actual importance of this relationship is beginning to be realized ${ }^{1}$
The birds view of health scenario is alarming, health needs are more demanding, the goal which aims at "Health for all" by 2000 A.D, which was initiated during 1977 (30 ${ }^{\text {th }}$ May 1977) by World Health Assembly. WHA decided to launch social, global target "Health for all by 2000 A.D" defined as attainment by all the people of the world, a level of health, which will enable or permit every individual to lead socially and economically a productive life" is tough task which is striving very hard to achieve the goal. As the health of the Indians is in the pangs complex causes which are inter-related these factors make the task more difficult but not an impossible one. Unless we go for the scientifically effective \& affordable strategies, it becomes a distant dream. ${ }^{2}$

Health care is tough task to country like India due to the pangs of poverty, illiteracy, ignorance \& conservatism. Public health care professionals are striving hard to focus more on prevention rather than on curative \& rehabilitative services as preventive services are simple, affordable, easy \& most suitable techniques of handling health care. Common practices like healthy life style, vaccination, sanitation, \& hygienic practices like use of handkerchief, balanced diet avoiding bare foot walking \& washing hands have proved that these practices greatly contribute to the healthy life. ${ }^{3}$

India is land of villages ,management of rural health care services is a tough task as the components of rural life and Problem associated with it are multi dimensional and complex in nature .Rural health care demands not merely infrastructure ,adequate trained staff or free drugs but more important than that is making people to utilize the available services 
and gain confidence in health care providers, which is not given prime importance $\mathrm{A}$ popular quote "Health is Wealth" and ancient 4 Chinese quote which stands ever green.

\section{"If the National plan is one year - Grow Rice If it is for 25 years -Grow Trees If it is for 100 years- Do Health Education"}

To have the proper strategies of community participation is a must. This can be achieved by creating awareness regarding health problems, ways to alleviate them \& channelize the efforts. In this contest the news papers play a major role. ${ }^{5}$ healthcare .Even a non formal health education for every one residing in a particular community can make miracle, A wide range of diseases can be prevented and can be treated at community level health care unit by the community itself, if the members of the community are having basic knowledge of health and disease, awareness about common community health problems and also if the members of community are trained to follow simple affordable, economical preventive measures to protect their family health can also greatly contribute to the community health which leads to national development

If awareness is created among general public the new Philosophy of health May be stated as.....Health is fundamental human right, It is the essence of productive life Is intersect oral, is an integral part of development, Is central to the concept of quality of life, It involves individuals, state ,national and international responsibility

Health care starts at homes and continues at schools, workplaces, neighborhoods, and communities. It is known fact that taking care of ourselves by eating well and staying active, not smoking, getting the recommended immunizations and screening tests, and seeing a doctor when we are sick all influence our health. Our health is also determined in part by access to social and economic opportunities; the resources and supports available in our homes, neighborhoods, and communities; the quality of our schooling; the safety of our workplaces; the cleanliness of our water, food, and air; and the nature of our social interactions and relationships. The conditions in which we live explain in part why some Americans are healthier than others and why Americans more generally are not as healthy as they could be. Healthy People 2020 highlights the importance of addressing the social determinants of health by including "Create social and physical environments that promote good health for all" as one of the four overarching goals for the decade. This emphasis is shared by the World Health Organization, whose Commission on Social Determinants of Health in 2008 published the report, Closing the gap in a generation: Health equity through action on the social determinants of health_The emphasis is also shared by other U.S. health initiatives such as the National Partnership for Action to End Health Disparities_and the National Prevention and Health Promotion Strategy.

The Social Determinants of Health topic area within Healthy People 2020 is designed to identify ways to create social and physical environments that promote good health for all. All Americans deserve an equal opportunity to make the choices that lead to good health. But to ensure that all Americans have that opportunity, advances are needed not only in health care but also in fields such as education, childcare, housing, business, law, media, community planning, transportation, and agriculture. Making these advances involves working together to:

- Explore how programs, practices, and policies in these areas affect the health of individuals, families, and communities.

- Establish common goals, complementary roles, and ongoing constructive relationships between the health sector and these areas.

- Maximize opportunities for collaboration among Federal-, state-, and local-level partners related to social determinants of heal ${ }^{6}$

Health services throughout world are now confronted with new challenges, they are no longer considered merely as a complex of solely medical measures but as an important component of socio economic development (WHO1975).

The literacy is the only tool which can help us to fight the battle "Health For All" there is utter need for health education for every one which is the Only key to break such vicious cycle ,and which an developing nation like India can afford \& adopt to reduce DALY (Disability Adjusted Life Years) is creating awareness among general public. There is need to focus more attention \& give priority for this dimension of health care. 
If a plan is to change health culture to attain health for all we need to understand the relations between community, medicine and behavioral science, these three terms are interdependent This article makes an attempt to specify the relationship briefly.

\section{Community and its changing structure}

Community always denotes "we feeling" or community sentiments as elements of community are group of families, locality and sentiments. Every community differs from other community in its language, mores, folkways, myths, superstitions, caste, class, marriages and family patterns etc. Interdependency is permanent feature of any community .In a community life a greater emphasis is laid on unity , which is due to common living and thinking members generally have common interest and they are in agreement with one another .

If we are committed to help a community improve its health we have to start with people as they are and with community as it is . Behavioral scientists opine that every community has a health culture of its own which is based on social, economical political religious and cultural factors existing in particular community

Change is the permanent and universal phenomena in the society. In the modern era one could see that in the process of social change community structure is changing due to the influence of multifaceted factors like Urbanization. Industrialization, Commercialization, Migration, Development of science and technology, Roads and Transportation including Globalization have initiated social, economic and cultural changes in the contemporary urban and rural communities. That is why it is essential for the health functionaries to know the emerging changes in the families or communities and also to understand status of community health, causes of sickness, it is necessary to know to know what sort of families it has ,how members of that community live, what food they eat, what is their occupation and income, their customs, beliefs, values ,traditions and habits as all these have impact on health of community ,understanding relationship between human life and social environment, helps health care service providers to provide need based comprehensive health services .

\section{Concept of Health and Diseases}

The concept of the terms Health and Disease are changing, understanding of health and disease is the basis of Health Care. Health is not perceived in the same way by all the members of community including various professional groups -Bio Medical scientists, Social scientists \& Health Administrators On one hand it is a highly personal responsibility \& on the other hand a major public concern . It thus involves the joint efforts of the whole social fabric viz the individual, the community, \& the state to PROTECT and PROMOTE health it may be stated as.....Health is fundamental human right, It is the essence of productive life, Is intersectorial, Is an integral part of development, Is central to the concept of quality of life, It involves Individuals, State, National and International responsibility

The concept of causation of disease has also shown global changes, as per Richardson (1945) professor of medicine progressed from the disease origin to the total personality of the patient as a member of family in its community setting .a five decade old opinion of Seal (1963),the concept of disease causation evolved by stages from supernatural and deistic origin to the natural and multi factorial causation .similarly Mahajan (1972)has emphasized that poor social and virulent is the main factor responsible for poor health or sickness .Keeping in view the changing modern concepts regarding disease causation many scientists have repeatedly pointed out the contribution of behavioral sciences to medical science.

\section{Community health behavior and behavioral sciences}

Park et.al (1979), the term behavioral science is applied to those discipline committed to the scientific examination of human behavior. Park has also elaborated that these sciences mainly Sociology Social psychology and Social Anthropology. As per Barelson (1967)these three disciples have their own history, their own focused areas of study and perspectives of approach each shares however a common concern to the study of human and community health behavior and a scientific commitment to account for it . It is a authentic fact that the health status of a community is influenced by cultural traits Seal (1963) Leonard (1952).Similarly there are numerous social factors which are also inter related and complexly linked with the individual and community health status . 
After independence Indian health administrator as brought out by Banarji (1975) directed their appeal particularly to words the then social scientists engaged in generating behavioral science knowledge to legitimize the existing community structure and social relations .The response was generous ,Eminent Social and behavioral scientists from the west Marriot (1955) and Lewis (1958)and the studies carried out by their Indian disciples like Hasan (1967) Dhilon (1971) Kakar (1977)and Prasad (1979) drew attention to the certain basic social and cultural factors which militate against acceptance of community health practices .

Behavioral sciences have grown considerably evolving a theoretical frame work pertaining to human health behavior, similarly Faster (1970)pointed out that for avoiding disastrous decisions in the field of community health, the knowledge of certain areas of behavioral science serve as an accurate map which every health professional should carefully go through, if he is not to get lost and which will also help him to know his ways around the community

The birds view of existing health care facilities and its utilization and poor participation of community members compel policy makers and health care professionals to find out why people are reluctant to utilize (free )service ? Why hostile towards acceptance of new health innovation? Why bias about self health care? What are the outlook on cause of the diseases and treatment? What prevents them to go for prevention of diseases ? for answering these questions public health workers are turning increasingly to those behavioral sciences whose central concern is human health behavior and conditions that determine it .Leonard opined that medicine public health are strongly and rapidly realizing that the specialized knowledge and techniques of behavioral science are required for the proper study of community health needs and their solutions, the above fact is further strengthened by Wahi (1992) that medicine is intrinsically and essentially a social science and as long as this fact is not recognized we shall not be able to enjoy its benefits .

It is also evident as pointed out by Calfman that behavioral sciences can suggest to the community health planners about what type of network of communication is likely to produce better results and for which program and among what type of people thus many community health problems are essentially social in nature and need social solution

In the field of community health needs the cooperation of and help of behavioral scientists in studying problems such as the social components of health and diseases ,behavior of people in illness ,efficient use of medical care and medical institutions from the above facts it is noteworthy that the physician and other community health care providers need two kinds of knowledge medical science knowledge and social science knowledge so that they could more effectively serve the patient as well as community

\section{Recommendations}

Now it is wake up time to India to fight against all odds and move towards health for all by reorienting existing priorities in health care services from curative to preventive on the basis of felt need services to the community. On the whole overnight we cannot change the health scenario, but slow and steady race of change in the health culture of people will definitely lead us to "Health for all" Medical social work is a powerful weapon to realize comprehensive and qualitative health care is used only in a few limited fields and considered as less important component

- Existing manpower has to be trained to be the catalyst to bring change in health culture

- Involvement of relevant partners for social action on social determinants at all levels

- - home, school and worksite,

- Grass root level introduction of concept of importance of social determents in health

- Apex body focusing on SDOH for Supportive supervision to maintain quality health care

- Poverty eradication and compulsory education have to be taken up to change health culture of community

- Instead of using Television use Tell a women to communicate health care message

- Priority should be to BCC \& IEC on preventive measures to curb communicable \& non communicable diseases.

- Alternative and affordable new technique to tackle social aspect of health problems should be initiated

- Medical education system has to focus more on inclusion of social science in the curriculum 
- Medical social worker have to be liaison between policy makers ,health care providers \& public to formulate need based health care activity

\section{References}

1. S C Gupta N C Chugh, Behavioral science and community health problems Swasth hind October 1986

2. Veena Algur et al Coverage of health issues in News papers an Observational study. International journal of current research and review oct 12 vol 4(19)p192

3. Veena S Algur, MC Yadavannavar Hand washing practices among patients attending RHTC International journal of health science and Research Vol 2 issue 2 May 2012 p-46

4. Swasth Hind March April 1998 p 69

5. Veena Algur et al Coverage of health issues in News papers an Observational study. International journal of current research and review oct 12 vol 4(19)p192

6. http://www.healthypeople.gov/2020/topicsobjectives/topic/social-determinants-health

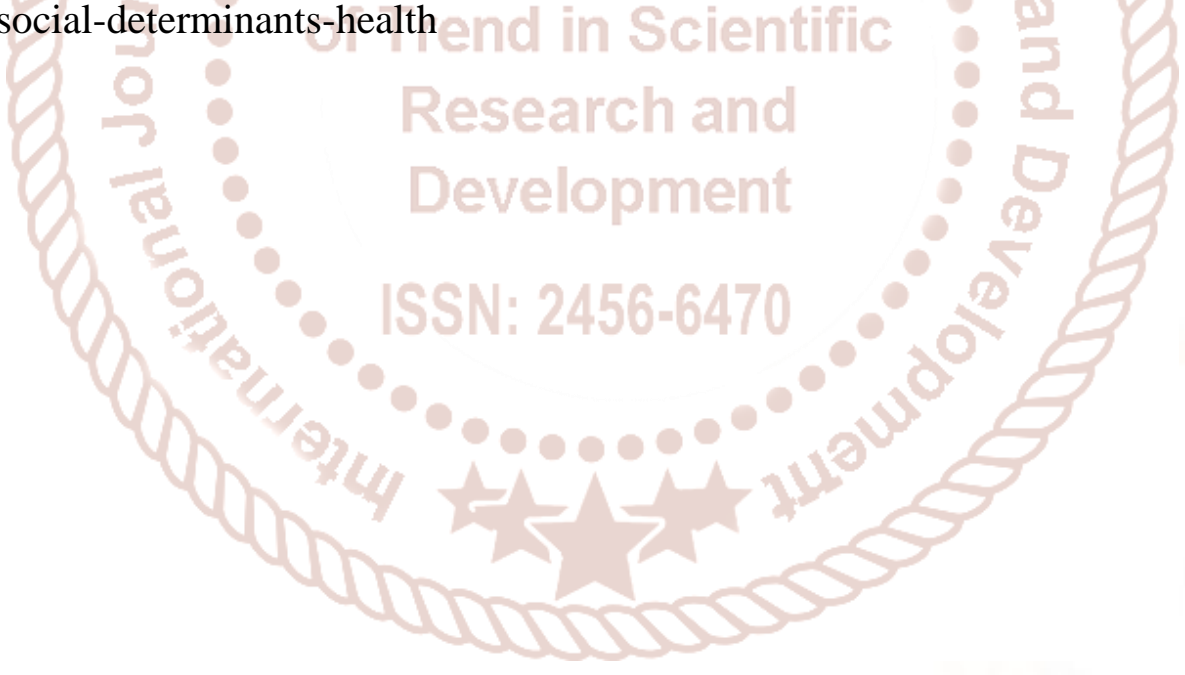

